\title{
Developing an Android App for Dementia Patient Location: Prevention of Wandering Case Study
}

\author{
Latifah Alraddadi, Jinan Fiaidhi and Sabah Mohammed \\ Department of Computer Science, Lakehead University, \\ Thunder Bay, Ontario P7B 5E1, Canada \\ \{lalradda, jfiaidhi, sabah.mohammed\}@lakeheadu.ca
}

\begin{abstract}
In dementia patients, wandering means acting on a desire to be elsewhere. Wandering can be dangerous. According to the Alzheimer's Association Safety Center Six in 10 people with dementia will wander and become lost; many do so repeatedly ${ }^{l}$. Most caregivers prefer to use tracking technologies as a back-up to other strategies of management, particularly supervision by a caregiver and locked doors. In cases where the risk of harm from getting lost to be low, tracking is used to preserve the independence of the patient with dementia. Various efforts have been attempted to attain a better understanding of mobility behavior of the dementia patients, but most studies are based on institutionalized patients and the assessment usually relies on reports of caregivers and institutional staff, using observational approaches, activity monitoring, or behavioral checklists. However, there are many current technologies that uses GPS devices to find the precise location on a map of a person equipped with these devices (e.g. bracelet). However, to date, research into dementia patient activities, using simple mobility tracking technologies has been largely limited to studies tracing of the routes of patients using the patient smart phone. The objective of this case study is to use the smart watch with the mobile phone along with messaging push technologies for alerting the patient and the caregiver and redirect the patient in case of wandering outside the prescribed region.
\end{abstract}

Keywords: Dementia, Location Aware Mobility, Smart Watch, Android, GPS Trucking

\section{Introduction}

In dementia patients, wandering means acting on a desire to be elsewhere. Some do indeed wander casually, mainly because they can't think of anything else to do-it just feels right to keep on moving. Not all wanderers do so aimlessly; some wander with resolve. Some patients wander to get away, when they feel that they are not at home, or there is something or someone bothering them. Some wander to reach a specific location - it may really exist, or just be imaginary ${ }^{2}$. Technology can assist in solving this problem where mobile phones in particular have been used for different purposes in dementia care, including memory enhancement and wandering safety [1]. Joea and Demirisa in 2013 [2] reviewed 510 articles published at PubMed and CINAHL. Findings from articles indicate that the use of mobile phones as an intervention can provide successful management for the dementia wandering problem. This article provide a practical setup of a technology that can use mobile phone along with smart watch to plan the possible dementia patient wandering region and to truck the patient movement and to guide the patient in case of wandering outside the planned region with push messaging technology.

\footnotetext{
${ }^{1}$ https://www.alz.org/national/documents/brochure_stayingsafe.pdf

${ }^{2} \mathrm{https} / / / w w w . a l z . o r g / c a c e n t r a l / d o c u m e n t s / S a f e t y \_49$-_Going_the_Extra_Mile_to_Prevent_Wandering.pdf
} 


\section{Related Research Work}

Several studies have piloted mobile technologies as an interventions for managing chronic conditions such as Alzheimer's/dementia. However, we can single some of the most related research work in trucking and guiding these type of patients. Lin et al in 2006 [3] describe a system that incorporates four types of technologies: radio frequency identification, geographic information system (GIS) and global positioning system. Although the proposed system help in searching the missing patients it require complex setting and experienced monitoring as it consists of a web service server, a database server, a message controller server, and a health-GIS (H-GIS) server. Patil et al in 2014 [4] integrate the Global Positioning System and Global System for Mobile Communication technology for real time people tracking using Google Maps. They used kind of embedded system technology programmed in two parts (tracking part and the displaying part). For tracking part, the embedded system is equipped with GPS, GSM modem and LPC 2138 (ARM) microcontroller for navigation purpose. The GPS provide information about the real time location of the people and sends the coordinate to the GSM modem through the microcontroller. This setting is even more complex to be used for trucking patients. Actually most of the other studies and systems that we found in the literature are designed for monitoring dementia patient for indoor activities $[5,6,7]$. These technologies are very complex to be used for monitor the outdoor patients activities. This case study attempt at developing a simple technology based on available mobile devices that any starter developer with knowledge of Android programming can set it for monitoring patients indoor and outdoor.

\section{Developing the Dementia Tracking Application}

The project setting is to incorporate two Android devices (Android Phone and a compatible Smart Watch). Android Studio was used as the development platform for designing and development of both mobile devices. Three elements of programming skills were required to integrate both of these devices along with some APIs like Google Maps:

a. FXML for designing the various user interfaces of the trucking system.

b. JAVA programming language is for backend components including the model and controllers of the trucking system and to communicating with server and the patient.

c. PHP programming language is for server side handling like developing webservices for android app and enabling android apps to communicate with each other.

Figure 1 illustrates the overall application structure. The trucking application include major components like the data server, the location service and variety of user and admin UIs. 


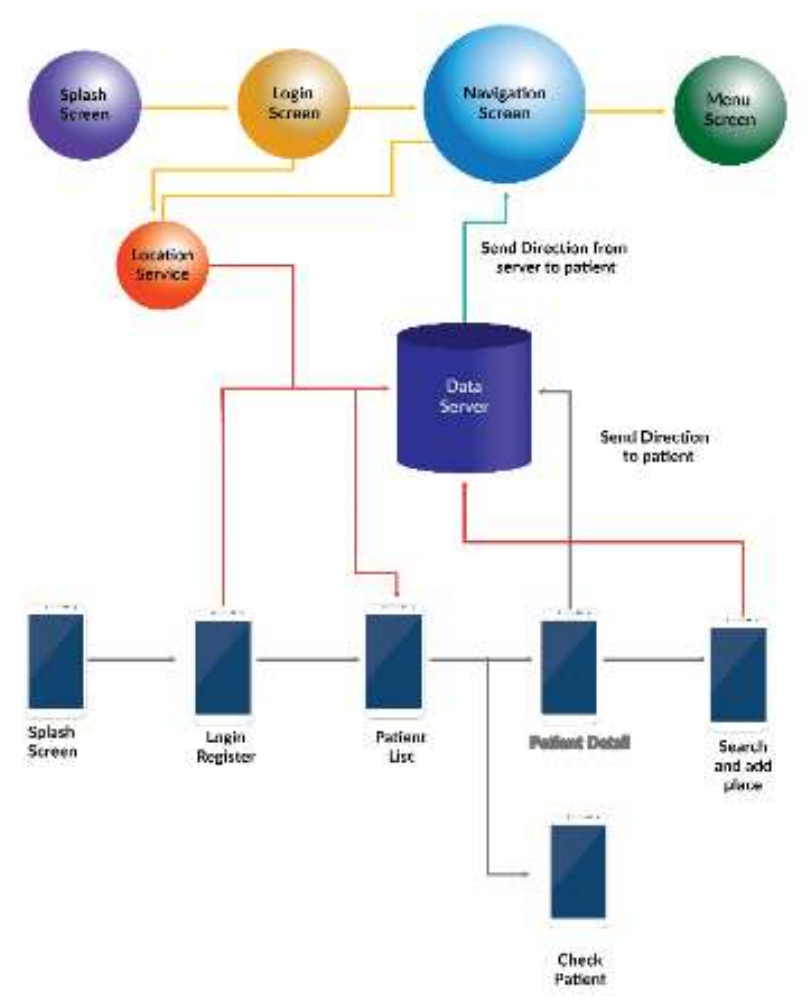

\section{Figure 1. The Overall Components of the Dementia Trucking mHealth System}

The functionalities of these components can be described as follows:

\section{User Registration}

This is a UI to register every patient at the trucking system (See Figure 2).



Figure 1. Registration Screens for both the Smart Watch and the
Mobile Phone

\section{The Help UI of the Dementia Patients}

In case the dementia patient requested help, the UI provide a notification and alert messaging that can be sent to the caregiver as well as the family members according to the notification setting. The notification component sends his real-time position to the selected members. The notification component is also able to direct the patient according to the replies received from the caregiver or the family member. For example if the patient would like help in finding the nearest coffee shop, the server will send the request to concerned members with the following information: 
a. Patient current location.

b. The place where patient wants to go.

c. The patient profile info.

Based on this information, the dementia patient will receive a route to his destination. However, the smart watch will continuously monitor the patient provided route and in case the patient followed a different route, the smart watch will be able to do the following (see Figure 3, Figure 4 and Figure 5):

a. Send a sound reminder asking the patient to look at the prescribed route on the smart watch.

b. Keep updating location of patient to the server so the others can find and truck the actual location of the patient.

c. Alerting the caregiver that the patient is lost after trying several times to direct him to follow the prescribed path,

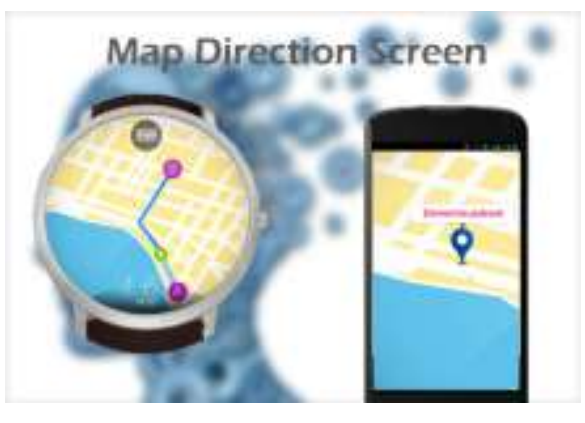

Figure 3. Directing Patient Using the Trucking System

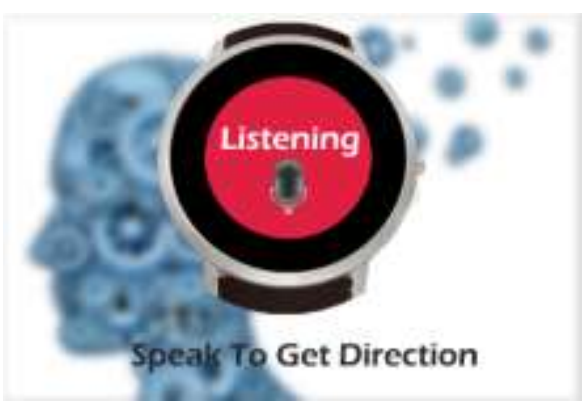

Figure 4. Listening to Patient Commands

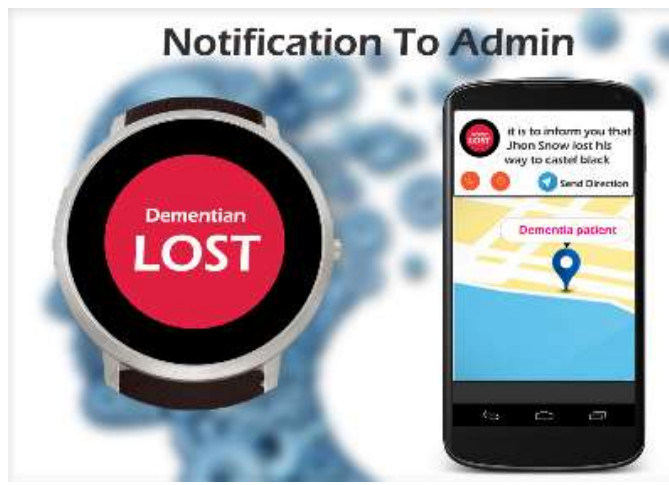

Figure 5. Alerting the Caregiver in Case of the Patient is Lost 
In this application, we used the Android LG Nexus 5 as the phone and the LG watch as the patient's tracking device. However, the phone need to accompany the patient so to maintain WiFi connection. To start the application the patient need to login process so the server can start trucking the patient location. The login page will generate a unique id for the patient (see Figure 5).
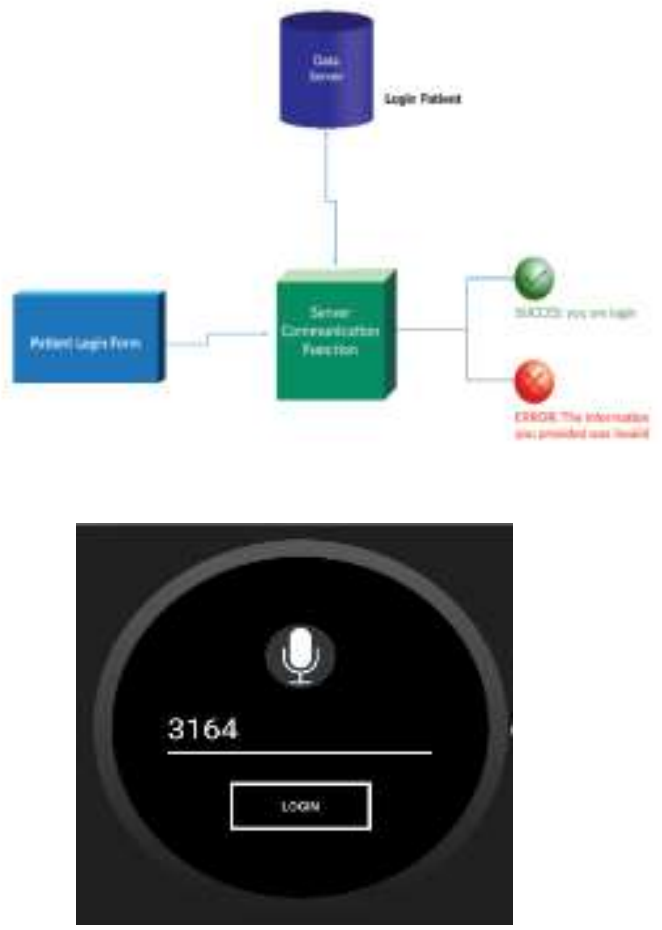

Figure 5 . Patient Registration Screen on Smart Watch

After a successfully login the system displays a tracking screen on the smart watch. The onMapReady get the current location of patient and display it on watch screen (see Figure 6).

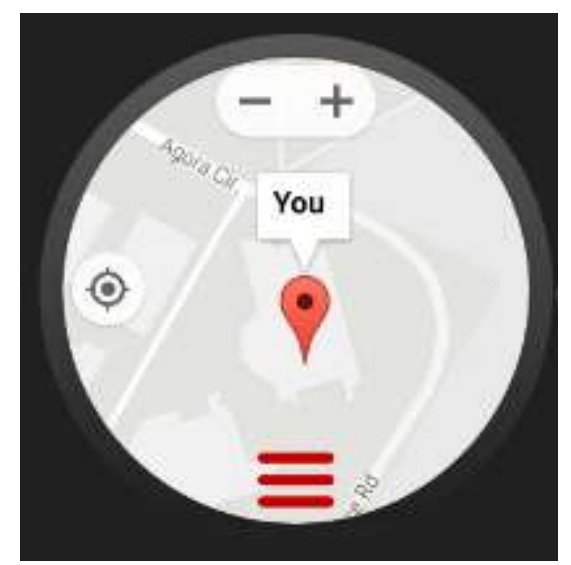

Figure 6. The Initial Patient Map

The listeners associated with this function listen to four different events:

- Location update

- New Direction received 
- Patient not following the route

- Patient Reached the destination

If location is updated, we move Google map camera to the new Location of patient so he can see it on map.

There is a listener registered in tracking screen for patient which listen to different events like

- Location update

- New Direction received

- Patient not following the route

- Patient Reached the destination

If location is updated, the Google API will be called to the update the location of the patient. However, the admin may ask the patient to go to a certain place (see Figure 7). In this case the system sends new direction notification to the user to go to this new place (see Figure 8).



Figure 7. The Address List Which Sent to Patient

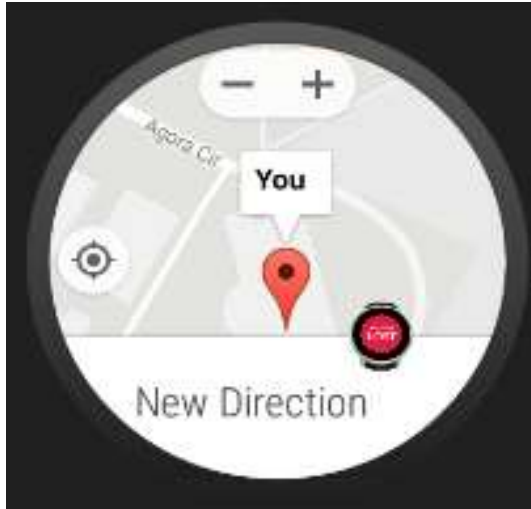

Figure 8. New Direction Received at the Smart Watch Screen

The complete flow of how notification work is illustrated in figure 9: 




Figure 9. The Message Flow for Directing the Patient to a New Direction

When new direction is received by the watch, a new event is fired to draw the new route. To draw new route a new function called getURL is called to get the direction to the new address from Google Maps (See Figure 10).

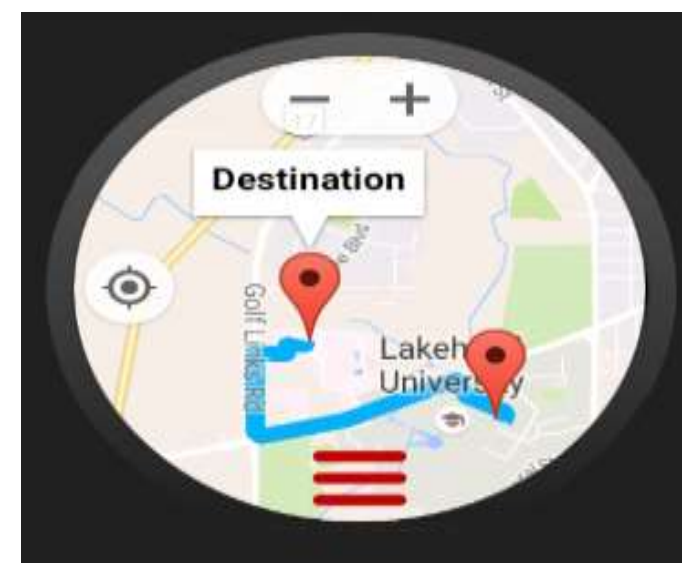

Figure 10. When the New Address is Received

If patient divert from the given route by $1 \mathrm{KM}$, the system sends an immediate textual notification to alert the patient to follow the prescribed route as displayed by the smart watch screen (see Figure 11).

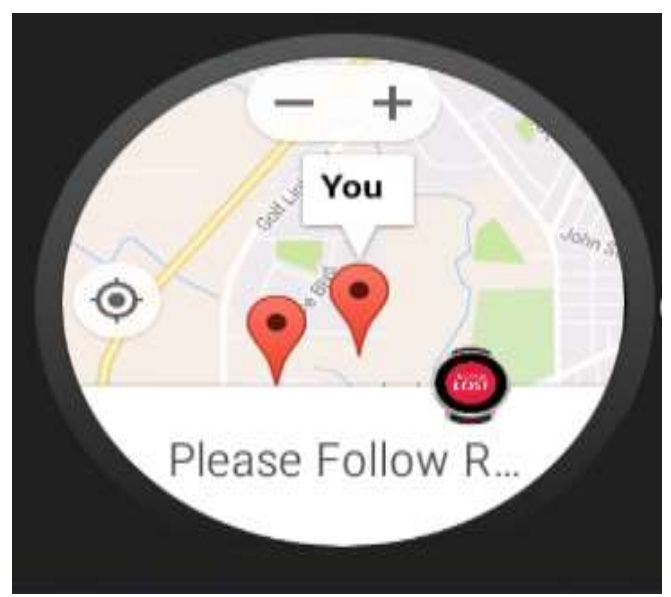

Figure 11. An Alert Notification asking the Patient to follow the Route 
Moreover, the patient can provide a response through his or her smart watch saying that he or she is lost (figure 12).

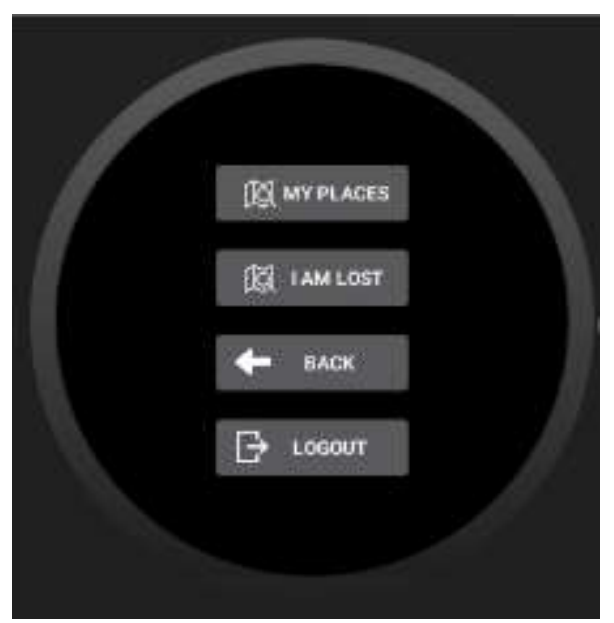

Figure 12. Options List for Patients

If patient is lost a notification with sound is also sent to the caregiver mobile (see Figure 13).

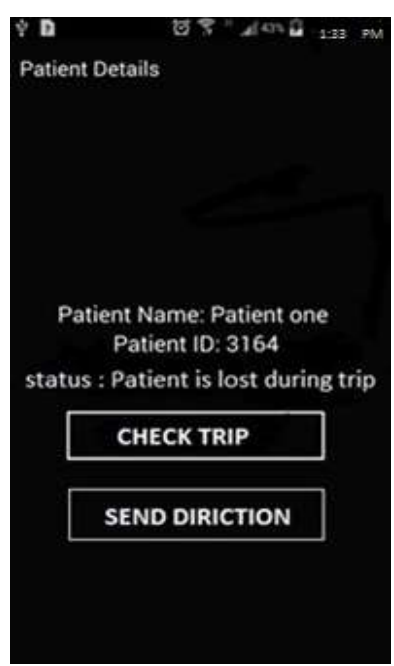

Figure 13. The Caregiver Patient Lost Alert

The caregiver can redirect the patient to follow the right route from by sending some new direction (See Figure 14). 


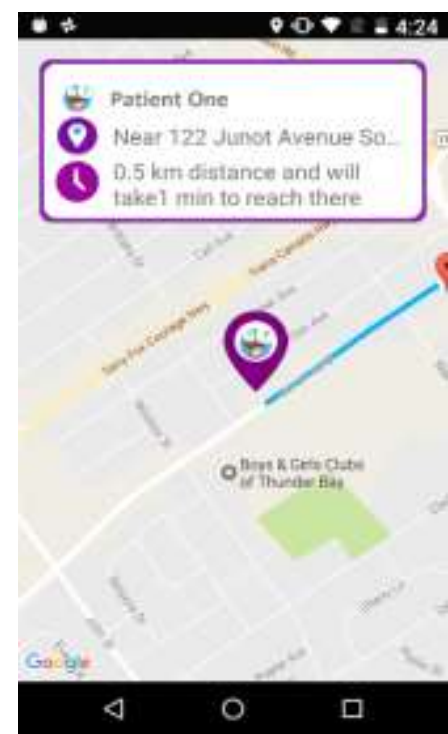

Figure 14. The Tracking Screen of the Caregiver

The caregiver can keep tracking the patient way by checking the trip any time, to make sure the patient is following the right path to reach to the desired distention. When patient reached destination, another notification is displayed (see Figure 15).

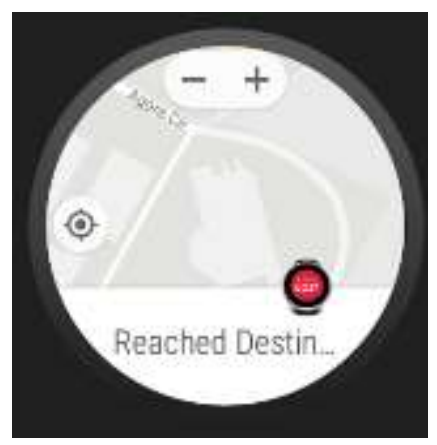

Figure 15. Patients Reached Destination Screen

The notifications between the caregiver and the patient helper to patient and patient has been achieved using the Google Cloud Messaging service (GCM). For this, we need to register our devices with GCM which return device token. This GCM token need to be sent to our server so we can get whenever we want to send notification to that device using device token. Figure 16 provide the notification flowchart. 


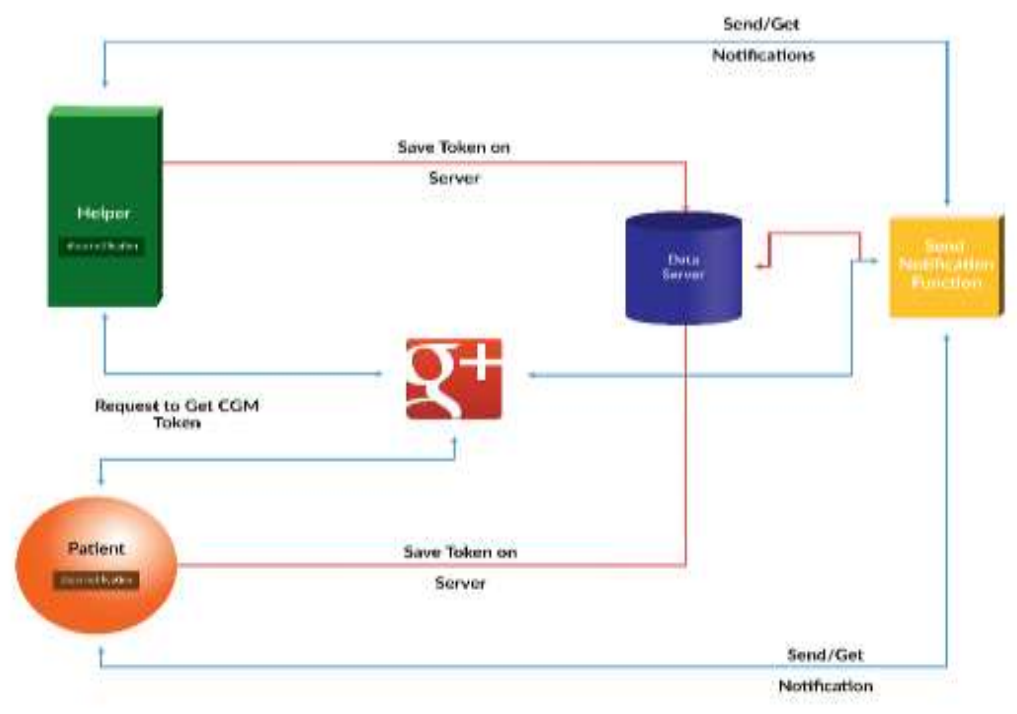

Figure 16. The Notification Flow Chart

\section{Conclusion}

In dementia patients, wandering means acting on a desire to be elsewhere. Wandering can be dangerous. According to the Alzheimer's Association Safety Center Six in 10 people with dementia will wander and become lost; many do so repeatedly ${ }^{3}$. Most caregivers prefer to use tracking technologies as a back-up to other strategies of management, particularly supervision by a caregiver and locked doors. In cases where the risk of harm from getting lost to be low, tracking is used to preserve the independence of the patient with dementia. Various efforts have been attempted to attain a better understanding of mobility behavior of the dementia patients, but most studies are based on institutionalized patients and the assessment usually relies on reports of caregivers and institutional staff, using observational approaches, activity monitoring, or behavioral checklists. However, there are many current technologies that uses GPS devices to find the precise location on a map of a person equipped with these devices (e.g. bracelet). However, to date, research into dementia patient activities, using simple mobility tracking technologies has been largely limited to studies tracing of the routes of patients using the patient smart phone. The objective of this case study is to use the smart watch with the mobile phone along with messaging push technologies for alerting the patient and the caregiver and redirect the patient in case of wandering outside the prescribed region. There are many implementation details was left as the authors were trying to describe the dementia trucking system. The detail work is described at the MSc dissertation of the first author.

\section{References}

[1] F. Miskelly, "Electronic tracking of patients with dementia and wandering using mobile phone technology", Oxford Age Ageing Journal, vol. 34, no. 5, (2005), pp. 497-499.

[2] J. Joea and G. Demirisa, "Older adults and mobile phones for health: A review", Journal of Biomedical Informatics, vol. 46, no. 5, (2013), pp. 947-954

[3] C-C. Lin , M-J. Chiu, C-C. Hsiao, R-G. Lee, and Y-S. Tsai. "Wireless health care service system for elderly with dementia", IEEE Transactions on Information Technology in Biomedicine, vol. 10, no. 4, (2006), pp. 696-704.

\footnotetext{
${ }^{3}$ https://www.alz.org/national/documents/brochure_stayingsafe.pdf
} 
[4] S.S. Patil,, D. P. Patil, and V. M. Davande. "Global Positioning System as a Safety Monitor for Alzheimer's Patients." International Journal of Emerging Engineering Research and Technology, vol. 2, no. 8, (2014), pp. 50-61.

[5] N. K. Vuong, S. Chan, and C. T. Lau. "mhealth sensors, techniques, and applications for managing wandering behavior of people with dementia: A review." Mobile Health, Springer International Publishing, (2015), pp. 11-42.

[6] T. Patterson, I. Cleland, P. J. Hartin, C. D. Nugent, N. D. Black, M. P. Donnelly, P. J. McCullagh, H. Zheng, and S. McDonough. "Home-Based Self-Management of Dementia: Closing the Loop", International Conference on Smart Homes and Health Telematics, Springer International Publishing, (2015), pp. 232-243.

[7] G. M. Revel, M. Arnesano, and F. Pietroni. "A low-cost sensor for real-time monitoring of indoor thermal comfort for ambient assisted living", Ambient Assisted Living, Springer International Publishing, (2014), pp. 3-12. 
International Journal of Bio-Science and Bio-Technology

Vol.9, No.3 (2017) 